\title{
THE OAK RIDGE ISOCHRONOUS CYCLOTRON: ENHANCEMENTS TO ORNL'S HRIBF DRIVER ACCELERATOR
}

\author{
B. Alan Tatum, James R. Beene, and Darryl T. Dowling, \\ Oak Ridge National Laboratory*, Oak Ridge, TN 37831, USA
}

\begin{abstract}
The Oak Ridge Isochronous Cyclotron, ORIC, serves as the driver accelerator for the production of radioactive ion beams (RIBs) at the Holifield Radioactive Ion Beam Facility (HRIBF). ORIC has served the physics community for almost 40 years as first a light ion accelerator, then as an energy booster for the Holifield Heavy Ion Research Facility's 25 MV tandem electrostatic accelerator, and now as a light ion driver for RIB production. In spite of its age, ORIC is a more durable and efficient accelerator than ever through extensive enhancements and development initiatives in recent years. Control system, power supply, central region, and mechanical upgrades have proven effective in making ORIC a key to recent research successes at HRIBF. This paper describes the ORIC enhancements and the recent RIB research to which it has contributed.
\end{abstract}

\section{HISTORICAL PERSPECTIVE}

The Oak Ridge Isochronous Cyclotron, ORIC, presently serves as the driver accelerator for the production of radioactive ion beams (RIBs) at the Holifield Radioactive Ion Beam Facility (HRIBF). However, the history of this variable energy, multi-particle, AVF cyclotron dates back over 40 years.

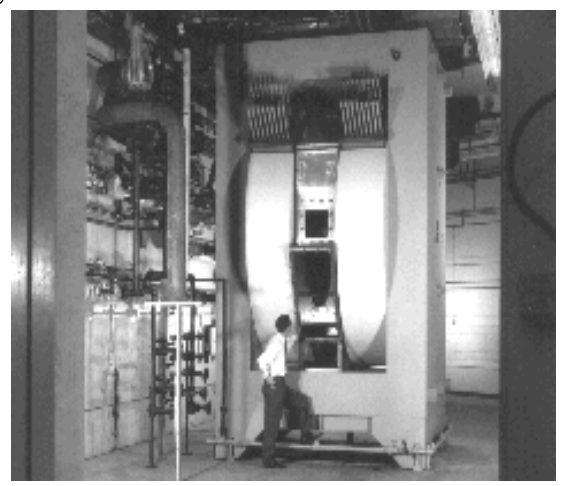

Figure 1: ORIC, circa 1960

Ground was broken for ORIC in October 1959, and the first internal beam was accelerated early in 1962. The first beam was extracted in 1963, and routine physics experiments with the extracted beam began in 1964 .

* Oak Ridge National Laboratory, operated by UT-Battelle, LLC, for the U.S. Department of Energy under contract number DE-AC05-00OR22725.
During the early years, ORIC produced light ion beams for medium energy nuclear physics research including proton beams with energies to $65 \mathrm{MeV}$, deuterons to 50 $\mathrm{MeV},{ }^{3} \mathrm{He}$ to $130 \mathrm{MeV}$, and alpha particles to $100 \mathrm{MeV}$. Beginning around 1967, ORIC was used to accelerate heavier ions, up to $\mathrm{A}=40$. The $25 \mathrm{MV}$ tandem electrostatic accelerator was added by 1981 for heavy ion acceleration up to A 90. ORIC was modified to serve as an energy booster for tandem beams as part of the new Holifield Heavy Ion Research Facility (HHIRF) and, in this coupled mode, ions up to $A=160$ were accelerated above the Coulomb barrier. Beginning in 1994, the HHIRF transitioned to its present configuration as the HRIBF for RIB research.

\section{ORIC AND HRIBF}

The HRIBF is an international user facility that produces beams of short-lived, unstable nuclei for research in nuclear structure and nuclear astrophysics [1]. The RIB production process begins with ORIC which is again used to produce high-intensity light ion beams (see Table 1) which are transported to an ISOL-type target/ ion source assembly [2] on a $300 \mathrm{kV}$ injector

Table 1: ORIC Beam Production

\begin{tabular}{|l|c|c|}
\hline \multicolumn{1}{|c|}{ Beam } & Energy & Max. Intensity on Target \\
\hline proton & $42 \mathrm{MeV}$ & $15 \mu \mathrm{A}$ \\
\hline deuteron & $50 \mathrm{MeV}$ & $12 \mu \mathrm{A}$ \\
\hline alpha & $85 \mathrm{MeV}$ & $3 \mu \mathrm{A}$ \\
\hline
\end{tabular}

platform. The reaction at the target produces radioactive atoms which are ionized in a high-temperature ion source, accelerated from the ion source at up to $60 \mathrm{keV}$, massseparated, transported off the injector platform at up to $300 \mathrm{keV}$, passed through a $\mathrm{m} / \Delta \mathrm{m}=20,000$ second-stage mass separator to filter out isobars, and injected into the $25 \mathrm{MV}$ tandem for final acceleration.

ORIC operated for approximately 1200 hours during FY 2000 and will do so again in FY 2001. Machine reliability has greatly improved due to a consistent program of accelerator system upgrades over the past 6 years. ORIC, once considered to be a weak link in the Holifield system due to excessive unscheduled maintenance, has become extremely reliable. Start-up times, historically on the order of two days, have now been reduced to 2 hours for previously accelerated beams. 


\section{ORIC ENHANCEMENTS}

Enhancements made to ORIC during recent years have contributed to operational successes, and have been focused on performance, reliability, and functionality. They consist of central region modifications for improved turn separation and ion source operability, investment in restoring original functionality, control system modernization, and power supply replacement.

\subsection{Ion Source and Central Region}

Substantial improvements have been made in cathode lifetimes and high-voltage rod reliability. The cathodes, cathode mounts, and water-cooled high voltage rods initially incorporated adjustments that allowed the gap between the cathode and the chimney aperture to be optimized. The tantalum cathodes were .15 " diameter and attached to the mounts by silver plated screw threads to allow disassembly and reuse of the mounts. Components were expensive to manufacture and cathode lifetimes were short, limited to 300 hours for protons, and less than 100 hours for alpha particles. Subsequent thermal analysis showed that the cathode tip temperatures were excessive and the majority of cathode erosion was due to evaporation. The cathodes and mounts were redesigned to increase thermal conduction by eliminating screw thread mounting and increasing the cathode diameter. New .25 " diameter cathodes are now cut to length from tantalum rod and pressed into the mounts. The simplified cathode mounts are inexpensive to fabricate and are expendable. The first set of cathodes operated for 768 hours (protons). The second set has over 450 hours and very little cathode erosion has occurred.

The water-cooled high voltage rod is a squirt-tube design. Initially, the rod tip consisted of a stainless steel sleeve furnace brazed to a tantalum rod tip, which was then brazed to the stainless steel squirt tube. The tantalum to stainless steel braze joint provided the water seal at the end of the high voltage rod and had to be vacuum tight. A combination of fabrication difficulties and mechanical stresses during cathode installation resulted in joint failures. The redesigned high voltage rods utilize a welded joint between the stainless steel tube and the stainless steel adapter sleeve to provide a vacuum tight joint for the water coolant system. A copper rod tip adapter is then brazed to the stainless steel adapter sleeve providing thermal conductance, but not a vacuum seal.

Attempts to improve extraction efficiency and turn separation have focused on improving transmission characteristics between the source chimney aperture and puller electrode by positioning them closer together and better matching the apertures. Initial tests have shown promise, with high-intensity proton extraction efficiencies improving from $55 \%$ to around $70 \%$.

\subsection{Functional Restoration}

Several functional aspects of ORIC have been unavailable in recent years, and an on-going effort is being made to restore its versatility. ORIC utilizes a set of ten concentric trimming coils mounted to the main magnet poles. Over the past 15 years, the two outer coils, T9 and $\mathrm{T} 10$, and one-third of coil T8 have become inoperable due to internal water leaks. Thus far, it has been possible to tune around these losses with some degradation of extraction efficiency and inability to isochronize certain beams. Loss of any additional coils would be devastating, so a new coil set was fabricated and is available for installation when needed.

ORIC was originally equipped with three sets of three each harmonic coils and one set of valley coils. The valley coils are now configured as a fourth set of harmonic coils, and only coil sets one and four have been available due to the lack of operable power supplies. To restore part of this capability, a set of new 250A harmonic supplies has been purchased and installed for use on either the number two or three coil sets as needed.

When ORIC was modified as an energy booster for the HHIRF, the rf system was reconfigured including the dee and liner assembly. The frequency range was shifted and now precludes the possibility of accelerating protons to energies above $50 \mathrm{MeV}$. Fabrication of a new dee/liner assembly is again being considered as part of the HRIBF upgrade plan because higher energy protons would in some cases more than double radioactive ion production.

\subsection{Control System}

ORIC controls, originally hardwired, have undergone a series of changes throughout the accelerator's lifetime. In recent years, the 1970's vintage Modcomp control computer was completely replaced. ORIC controls are now predominantly digital and distributed. Allen-Bradley programmable logic controllers (PLCs) are used exclusively for both discrete and analog I/O. The newer, large power supplies have been purchased with on-board PLCs and are networked on ControlNet for deterministic I/O and on Ethernet for operator interface, programming, and diagnostic purposes.

When the HRIBF was constructed and ORIC controls modernized, Vsystem, by Vista Control Systems, was chosen as the standard for unifying all HRIBF controls. ORIC, new beam lines, the RIB injector, and the tandem accelerator controls were converted to Vsystem. As the facility developed, it became evident that the EPICS control system was a more appropriate long-term choice [3]. The tandem and tandem beam lines now operate under EPICS, the RIB injector and injector beam line will be converted in the near future, and ORIC will be converted last. The order of conversion has depended on issues such as Vsystem performance under UNIX, and the RIB Injector dependence on the now obsolete VAX/VMS 
to VAX/ELN interface. ORIC's VAX/VMS-to-PLC Vsystem implementation operates quite reliably for now, but VAX/VMS is not a long-term option so the conversion to EPICS will inevitably take place.

\subsection{Power Supply Replacement}

Numerous ORIC power supplies from the original installation in the early 1960's continued to be used well into the 1990's. However, most began to experience chronic instabilities in ripple and regulation, and failure of various obsolete components resulted in excessive interruptions to operation. Most of these power supplies have now been replaced. In particular, eight new 800A trim coil power supplies have been installed along with a new 6000A coaxial extraction channel supply, and a 3000A lower magnetic extraction channel supply. The 1938 vintage motor-generator set that powers the main magnet coils remains the single greatest vulnerability. This $5000 \mathrm{hp}$ motor and $1750 \mathrm{kVA}$ generator provides up to $5000 \mathrm{Adc}$ at $360 \mathrm{~V}$ and, although it continues to run well, some of its windings have begun to separate. Thus it is likely that it will be replaced with a conventional power supply in the next few years.

\section{RADIOACTIVE ION BEAM RESEARCH}

Bringing the HRIBF on-line as a user facility for RIB research has required enormous time and effort, but it has paid off over the last two years as a remarkably productive period of RIB research has commenced. The HRIBF beam development program has been physics driven, initially focussing on fluorine beams based on astrophysics community interest in ${ }^{17,18} \mathrm{~F}$. To this end, HRIBF's Advanced Projects R\&D Group developed a novel target system, based on thin refractory oxide fibers for the ${ }^{16} \mathrm{O}(\mathrm{d}, \mathrm{n}){ }^{17} \mathrm{~F}$ and ${ }^{16} \mathrm{O}\left({ }^{4} \mathrm{He}, \mathrm{pn}\right){ }^{18} \mathrm{~F}$ production reactions, along with a completely new concept in ion sources called the Kinetic Ejection Negative Ion Source (KENIS)[4]. This system has proved remarkably effective and robust, allowing HRIBF researchers to complete successful experiments with ${ }^{17,18} \mathrm{~F}$ beams of intensities up to $10^{7}$ ions $\mathrm{s}^{-1}$. These included a successful search for the long-sought and astrophysically important $3^{+}$resonance in ${ }^{18} \mathrm{Ne}$ using the ${ }^{17} \mathrm{~F}(\mathrm{p}, \mathrm{p})$ reaction [5], and subsequent nuclear astrophysics studies using ${ }^{17} \mathrm{~F}(\mathrm{p}, \alpha){ }^{14} \mathrm{O}$, ${ }^{18} \mathrm{~F}(\mathrm{p}, \mathrm{p})$ and ${ }^{18} \mathrm{~F}(\mathrm{p}, \alpha){ }^{15} \mathrm{O}$, producing unique, high-quality excitation function data $[6,7]$. The ${ }^{17} \mathrm{~F}$ beam was also used in a search for di-proton decay from low-lying resonances in ${ }^{18} \mathrm{Ne}$, which produced the first definitive evidence for the simultaneous emission of two protons [8]. Evidence for this exotic decay mode has been sought since the 1960s. Recently, accelerated neutron-rich RIBs have been generated by proton-induced fission of ${ }^{238} \mathrm{U}$. These RIBs were produced in highly permeable targets of uranium carbide deposited on open-format carbon matrices [9]. Over 100 neutron-rich radioactive species with intensities $>10^{3}$ ions $\mathrm{s}^{-1}$ have been produced. Coulomb excitation measurements have been made on at least six radioactive species in the mass 130 region, and a test run for fusion-evaporation studies with neutron-rich RIBs has also been completed.

These remarkably successful campaigns with both neutron rich and neutron deficient RIBs are a product of the intense efforts of HRIBF staff during recent years to adapt existing accelerator facilities to RIB production, developing novel target and ion source systems, and assembling a world-class suite of experimental equipment carefully optimized for RIB experiments.

\section{REFERENCES}

[1] Tatum, B.A., et al. "Development of the Holifield Radioactive Ion Beam Facility," Proc. Part. Accel. Conf., Vancouver, B.C., Canada, Am. Phys. Soc., May, 1997.

[2] Stracener, D.W., et al. "Ion Sources Used In The Production Of Radioactive Ion Beams At The HRIBF," Fifth Int'l Conf. on Physics with Radioactive Ion Beams (ISOL'01), Oak Ridge, TN, March 2001.

[3] Sinclair, J.W. "HRIBF Control System Upgrade Using EPICS," Symposium of North Eastern Accelerator Personnel, Knoxville, TN, World Scientific, Oct. 1999.

[4] Alton, G.D., et al. 2001. "A New Concept KineticEjection Negative-Ion Source for RIB Generation," Fifth Int'l Conf. on Physics with Radioactive Ion Beams (ISOL'01), Oak Ridge, TN, March 2001.

[5] Bardayan, D.W., et al. "Observation of the Astrophysically Important $3^{+}$State in ${ }^{18} \mathrm{Ne}$ via Elastic Scattering of a Radioactive ${ }^{17} \mathrm{~F}$ Beam from ${ }^{1} \mathrm{H}$," Phys. Rev. Lett., vol.83, 1999 pp.45-48 .

[6] Blackmon, J.C., et al. "Measurement of the ${ }^{1} \mathrm{H}\left({ }^{17} \mathrm{~F}, \alpha\right){ }^{14} \mathrm{O}$ Cross Section Using a Radioactive ${ }^{17} \mathrm{~F}$ Beam at HRIBF," Mtg of Am. Phys. Soc., SE Section, Chapel Hill, NC, Bull. Am. Phys. Soc., Nov., 1999.

[7] Bardayan, D.W., et al. "Destruction of ${ }^{18} \mathrm{~F}$ via ${ }^{18} \mathrm{~F}(\mathrm{p}$, $\alpha)^{15} \mathrm{O}$ Burning Through the Ec.m. $=665 \mathrm{keV}$ Resonance," Phys. Rev. C., 2001.

[8] Gomez del Campo, J. et al, Phys. Rev. Let., 86, 43 (2001).

[9] Stracener, D.W., et al. "Radioactive Ion Beams from Uranium Carbide Targets at HRIBF," Annual Fall Meeting of the American Physical Society/Division of Nuclear Physics, Williamsburg, VA, APS, Oct. 2000 . 